\title{
Method for Deep Drawing Process Control Using Segmented-Multiple Active Drawbeads
}

\author{
Catalina Maier ${ }^{1, a}$, Viorel Paunoiu ${ }^{1}$, Vasile Marinescu ${ }^{1}$ and Alexandru Epureanu ${ }^{1}$ \\ ${ }^{1}$ Dunarea de Jos University of Galati, Manufacturing Engineering Department, 111 Domneasca Street 800201 Galati, Romania
}

\begin{abstract}
This paper presents a new method for deep drawing process control. The new method consists in: $i$ ) using of a segmented-multiple moving drawbeads; ii) imposing an optimal reference trajectory for drawbeads position; iii) in-process control where controlled variable is chosen the amplitude of deviation $\delta$ of the actual instant workpiece surface geometry with respect to the surface geometry during simulation and as manipulated variable is chosen the deviation $\Delta h_{D B}$ of the actual drawbeads position with respect to the programmed one; iv) periodical validation, calibration and updatation both of the control model and reference trajectory of drawbeads position; $v$ ) using a reduced order model of the complex system design. An experimental device conception and testing is presented. The results obtained using this device confirms the validity of the new proposed method for deep drawing process control.
\end{abstract}

\section{Introduction}

Sheet metal forming is one of the primary manufacturing processes which are continuously under new developments. The deep drawn process produces highstrength, lightweight parts more cost-effectively than other methods. Among the advantages offered by deep drawing are: rapid press cycle times, fewer operations required to finish a part, the ability to create complex geometries unattainable through other processes.

The performance in manufacturing of complex parts by thin sheet metal forming is crucial affected by the risk that the material strain-stress state, during deformation process, to exceed the allowable limits and consequently to appear the material fracture or wrinkling.

The sheet metal deep drawing technology is one of the most challenging processes in manufacturing. The size, shape, thickness and deep drawn metal used to produces sheet metal deep drawn part cover a diverse range of variable. Individual variables should be evaluated carefully in order to determine the optimum manufacturing method. Criteria of this optimum take account three quality considerations [1]: i) formability (wrinkling due to excessive compression and tearing because of high local tensile stress that cause thinning and failure); ii) dimensional accuracy (springback caused by elastic recovery); iii) consistency, that represent, minimizing dimensional variations due to the variation of different parameters (lubrication, material properties, thickness).

The new challenges in this field are: $i$ ) the use of new materials, and ii) a new concept of metal forming like an

\footnotetext{
${ }^{\mathrm{a}}$ Corresponding author: catalina.maier@ugal.ro
}

intelligent next generation technology considering user needs and future technology [2].

The new tendencies in design, optimization and control of the metal forming process determined the need of using new modelling techniques [3-8]. The main characteristic of the new models is their very short response time, this being the reason why they can be used for real-time control of the process. By analogy with the finite element method the new developed modelling techniques are called metamodelling [6], reduce order modelling [8] or macromodelling of the deep drawing process [9]. The idea of reduce order modelling is to use two models for optimization: a fine model (finite element model) of the process which has high accuracy with computationally expensive to solve and a reduced order model associated with the adaptive space mapping, fast to solve but less accurate.

This all considerations determine the motivation for researchers to continue those studies.

In the context of the work conducted in this field by different researchers $[1,10]$, the present paper propose a new method to control deep drawing process by using segmented-multiple active drawbeads and using a combination of different process control procedures.

The study consider a new device for a reduce order model of the deep drawing process and experimental and numerical results are presented.

\section{Description of the method}

The new method tries to improve significantly deep drawing process control considering following 
requirements: $i$ ) selection of the correct process variables in order to monitor real state of the material during the process and command the correction; ii) reduce time consumption for deep drawing process and control system design by reduced order modelling; iii) generation of accurate reference trajectories for the process variables control using FEA and experiments; $i v$ ) combine different process control procedures and use those principal capabilities.

In this context, the method's steps are:

- first step - we establish the process variables for complex deep-drawing process;
- second step consist in the design of the reduce order model of the deep drawing process;

- third step represents the identification of the reference trajectories and control models, using finite element simulation;

- the last step in this algorithm (figure 1), consisting in reference trajectories and control models validation it is optional and is applied only for one deep drawn piece belongs to a dimensional group; the decision point selects, function on the answer YES/NO to the question "is it the first piece for the dimensional group?", if we proceed or no the experimental validation.

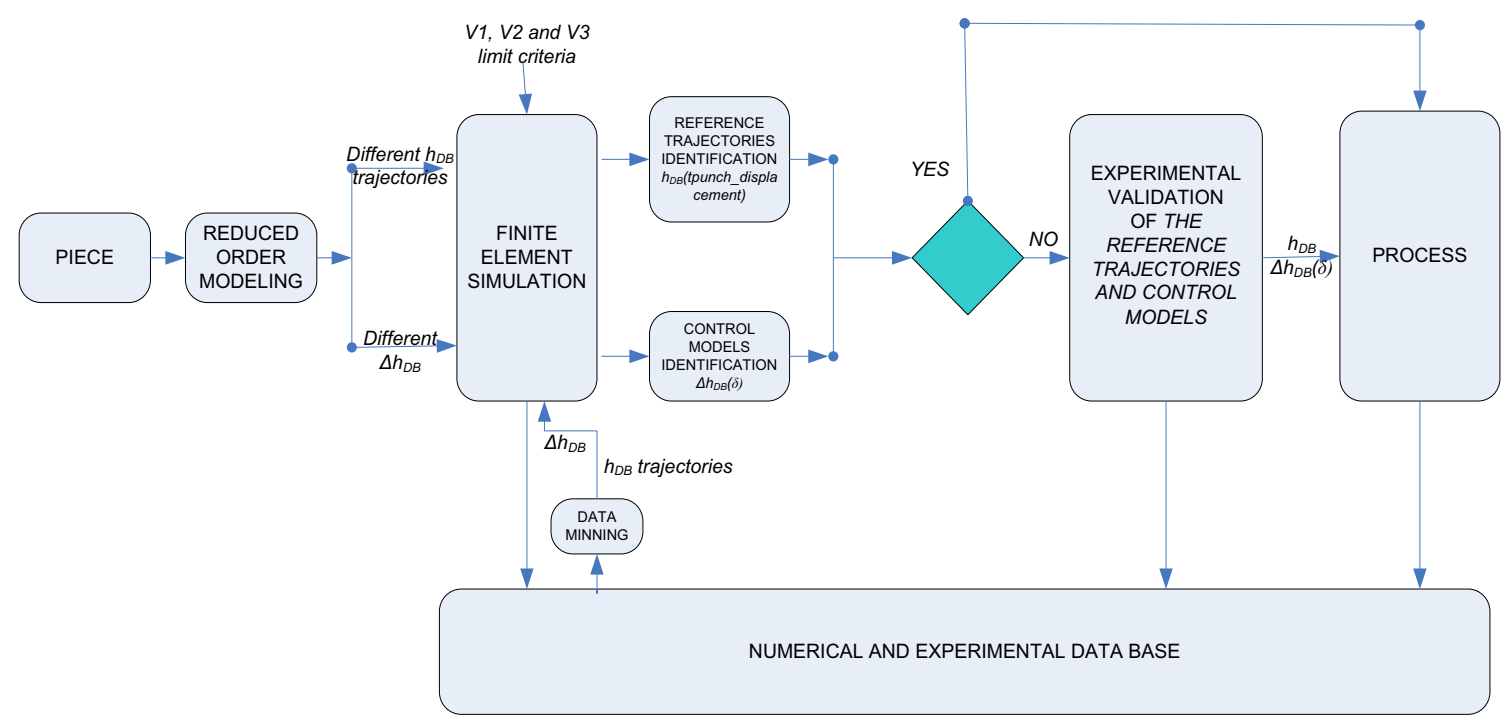

Figure 1. Algorithm of the new control process method

\subsection{Process variables}

The ideal in-process control of complex deep drawn would be the stress field control throughout the sheet metal based on cases presented in figure 2 , where $\sigma_{11}$ and $\sigma_{22}$ represent Cauchy stress tensor components. The limit values of $\sigma_{22} / \sigma_{11}$ stress ratio, respectively V1, V2 and $\mathrm{V} 3$, depend on the material properties and represent the criteria to define the following stress state types:

- stress state 1 - tension+compression with compression dominance determining wrinkles during the process;

- stress state 2 - tension+compression with tension dominance, determining wrinkles after the process, due to the springback;

- stress state 3 - biaxial tension when tearing appears.

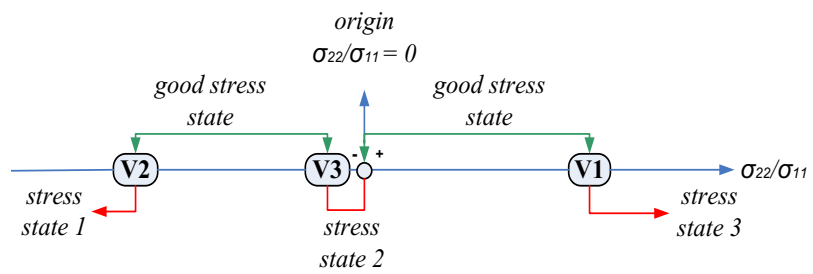

Figure 2. Characteristic stress states during complex deep drawing process
The good stress states (figure 2) represent the objective of the new method in order to respect quality considerations - formability, dimensional accuracy and consistency - presented at the beginning of this paper.

\subsection{Reduced order modelling}

In order to reduce time consumption for deep drawing process and control system design a reduce order model of the deep drawing process is applied. The main idea is to decompose complex deep drawing process in macroelements - elementary deep drawing processes and in their identification. Each one of these macroelements represents a particular case of the generalized elementary deep drawing process. The steps of real deep drawing process decomposition consists in: $i$ ) the delimitation of each elementary process and ii) his identification starting to a generalized elementary deep drawing process in order to establish its characteristics. The resulted process macromodel is less time consuming in comparison with finite element model, assures a very good precision and he is easier to use for the real-time control of deep drawing process [9].

The macromodelling of deep drawing process of any part provides new macroelements for enrich the numerical and experimental database. The decomposition of every real deep drawing process can use this database in order to build its macromodel. Each macromodel is 
characterized by: dimensions, boundaries conditions, reference trajectories, control model. For a large number of macroelements the finite element simulation is performed and the information obtained is also stored in the database.

\subsection{Reference trajectories identification}

Therefore, for each of the macroelement in the deep drawing process of the considered piece, from database is selected a situation that is the closest, so far, to the macroelement dimensions, new possible reference trajectories for process parameters are added and, finally FEM simulation is performed.

Analyzing the results obtained on the evolution of stress ratio and considering the limit criteria V1, $\mathrm{V} 2$ and $\mathrm{V} 3$, the optimal reference trajectory is determined. For example, FEM analysis of macroelement with a nonlinear contour was performing in terms of the following various trajectories (figure 3 ) of the punch and drawbeads:

- successive action of drawbeads (penetration up to a predefined value and maintain this position until the end of the process) and punch (whose action starts when reaching preset value the depth of drawbeads penetration), considered classical evolution used in deep drawing process;

- simultaneous action of drawbeads and punch with consideration of the various trajectories of drawbeads position.

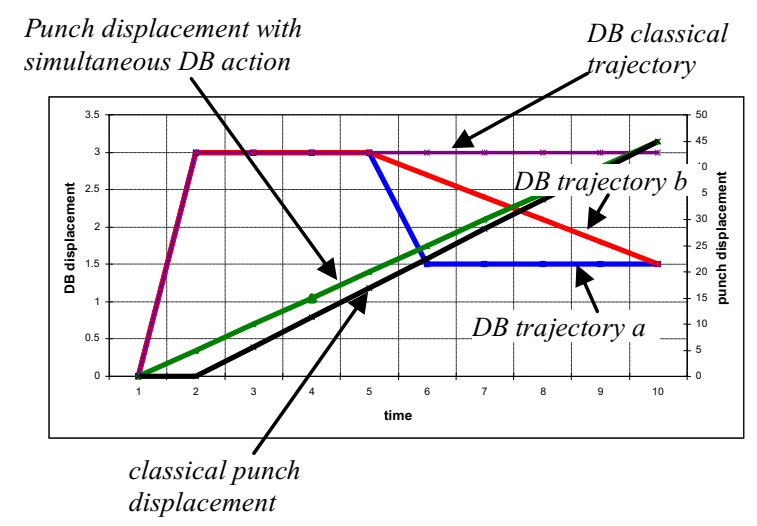

Figure 3. Different trajectories of drawbeads and punch

Numerical simulation results show a differential evolution of the ratio $\sigma_{22} / \sigma_{11}$ during the forming process, depending on the type of action (simultaneous or

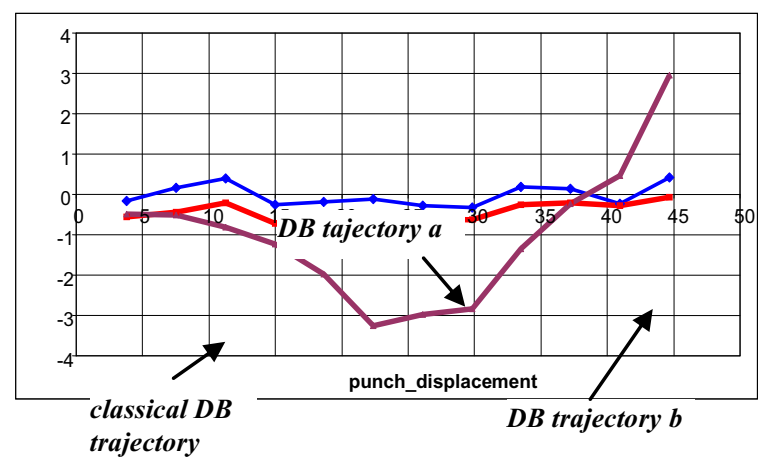

successive) of the drawbeads and punch and its reference trajectory (figure 4).

Figure 4. Stress state evolution function drawbead trajectory

From this analysis resulted that in classic case, used so far in deep-drawing processes while the drawbead enters into the material before the punch displacement starts and remains in this position until the end of the process, there is a substantial critical values exceeding 1.5 of the stress ratio that vary in this case between 2.94742 and -3.26625 .

In contrast, when simultaneous action of the punch and drawbead and gradual withdrawal after $50 \%$ of the active trajectory of the punch, the stress ratio is between 0.23 and 0.39 in the case of $D B$ trajectory $a$ and between -0.22 and -0.72 , if the $D B$ trajectory $b$ case is considered. Because the $D B$ trajectory a determinate the stress state 2 that can induce wrinkles formation after metal forming process (caused by springback), it follows that the drawbead optimal trajectory is $b$ which meet the limit criteria V1, V2 and V3 and also offers a reserve to the stress ratio variation before the loss of material stability.

We demonstrate that among various possible trajectories of drawbead position, we can determine one to meet the limit criteria V1, V2 and V3 and is considered the optimal reference trajectory.

\subsection{Control model determination}

As previously shown, process variable which will be controlled and particularly will have zero value is $\delta$. This variable is the deviation of real surface of the part from the surface obtained through numerical simulation. If the stress state, characterized by the ratio $\sigma_{22} / \sigma_{11}$, is becoming favourable to wrinkles formation, then variable $\delta$ will be nonzero and equal with deviation of the current surface of the part from the reference surface generated through numerical simulation. The correction of this deviation is done by a supplementary displacement of the drawbeads, $\Delta h_{D B}$, added to the programmed trajectory of its position. If a new deviation $\delta$ is recorded during deformation process, a new supplementary displacement will be given. The control model of the deformation process will be given by the function $\Delta h_{D B}(\delta)$. This relation is determined through numerical simulation with supplementary displacements at different steps along draw beads trajectory.

Finally, this information is transferred to the forming process carrying out an off-line control through programming of the reference trajectories and an on-line control through control models involvement.

A numerical and experimental database is created using the results obtained by numerical simulation, experimental research and from measurements and observations during real processes. It is constantly updated by data obtained from the execution of the actual metal forming process of complex parts.

Exploiting numerical and experimental database is performed by knowledge extraction techniques with aim of updating continuously both the reference trajectories and the process control model. 


\subsection{Reference trajectories and control model validation}

We chose between macroelements one that characterizes the most complete the process and the complex stress and strain evolution in the sheet. The macroelement who respect these conditions correspond to the nonlinear contour shape and include drawbeads. In our conception this macroelement represent the reduced order model of the real complex deep drawing process.

It is used to: $i$ ) validate the proposed method, ii) design and validation of metal forming process control model; iii) updatation of the database. Using the reduced order model of the complex deep-drawing process lead to reduced time required to its design and control as well as reduced costs for die try out procedure.

The conception of the device used, in this paper, to validate the new method is based on the: $i$ ) reduced order model of the complex deep drawing process; ii) different solutions used by researchers [11 - 15]; iii) functions to be assessed - control and monitor of the process variables $h_{D B}$ and $\delta$.

The components of the new proposed device are: $i)$ controllable metal forming device (figure 5), ii) gear mechanism with ball screws for the control of drawbeds; iii) monitoring and controling system of the manipulated process variables - programmed value $h_{D B}$ and its deviation $\Delta h_{D B}$ (monitoring and control system of metal forming process using a programable logic controller); $i v$ ) optical system measurement with machine vision algorithms.

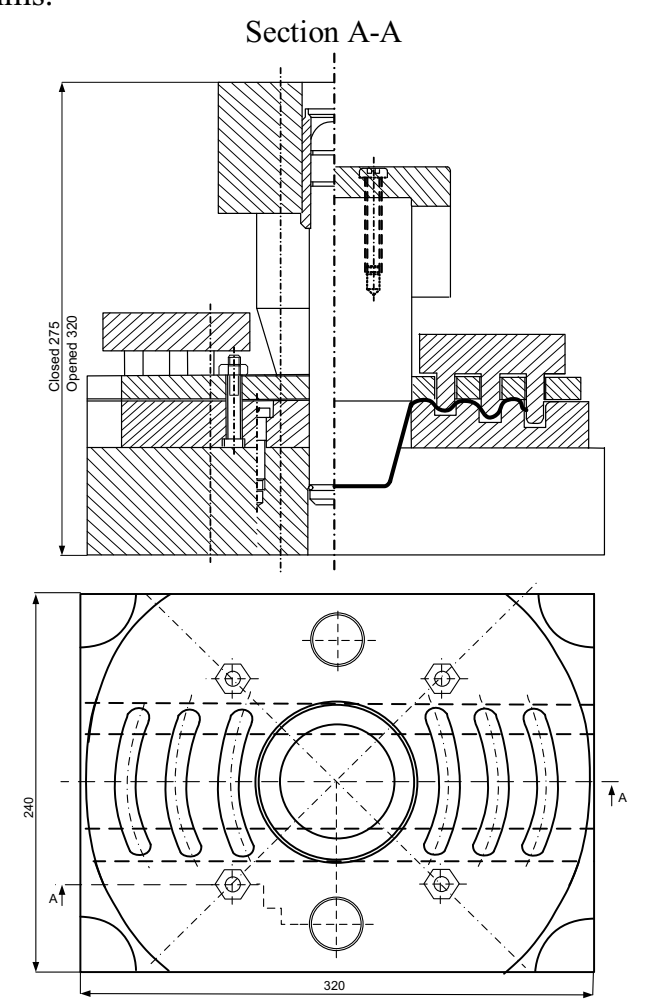

Figure 5. Controllable metal forming device

Physical simulation of graded action of the drawbeds, considering the results of FE analysis as above described, was performed considering two different construction of the drawbeds: multiple and single drawbeds. The use of multiple drawbeds in the methodology proposed in this paper is different from its use proposed by Papaioanu A, Schleich [12, 13], D. Vlahovic [16, 17] and Liewald M. R. [18] in that it is active (acting simultaneously with the punch and its position can be adjusted depending on the evolution of monitored parameter $\delta$ ) and its application is generalized for complex shape of parts.

\section{Results and conclusions}

Experimental researches are performed using the device previously presented, considering different thickness of the blank $(0.4 ; 0.6 ; 0.8 ; 1 \mathrm{~mm})$ and conditions of the forming process development (drwbeads maximal penetration, drawbeads and punch reference trajectories identified after the study presented in the chapter 2.3). In figure 6 we present the pieces obtained following these 3 conditions:

a). classical trajectory of drawbeads - penetration up to a predefined value and maintain this position until the end of the process - without in-process control (figure 6.a) b). reference drawbeads trajectory b, considered optimal after FE analysis, without in-process control (figure 6.b); c). reference drawbeads trajectory $b$, considered optimal after FE analysis, with in-process control (figure 6.c);

Maximal programed value of drawbeads penetration is $2 \mathrm{~mm}$ for a blank thickness of $0.4 \mathrm{~mm}$. It results the following observations :

- piece $\boldsymbol{a}$ presents wrinkles in the uncontrollable deformed zone during the process and large springback, after the finish of the forming process, in flange zone ;

- $\quad$ piece $\boldsymbol{b}$ presents a small level of the wrinkles in the uncontrollable deformed zone during the process and accepted springback of the flange after the forming process finish ;

- piece $c$ results without wrinkles during the process and springback of the flange after the finish of the forming process.

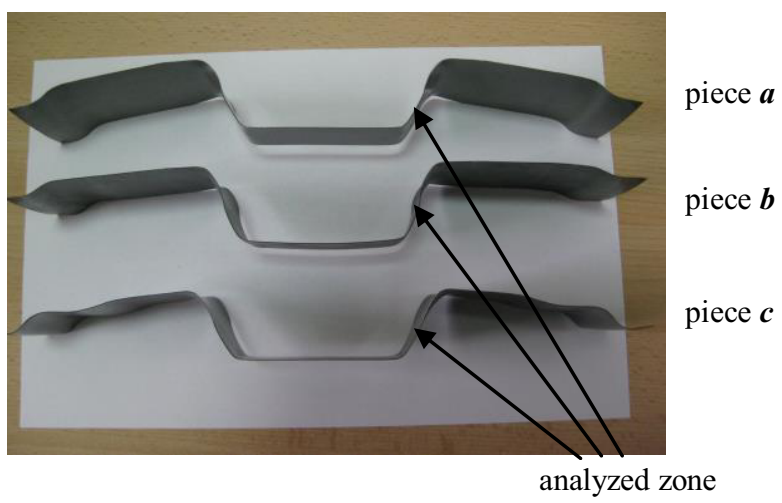

Figure 6. Experimental results

We analyze the deformed shape in the central zone of the free deformable part of the specimen, where the instability of the material is highest, for the considered conditions, as above. This instability is due to the uncontact conditions between the specimen and the tools. In figure below is presented the deformed shape at the end of the process for the studied cases. 


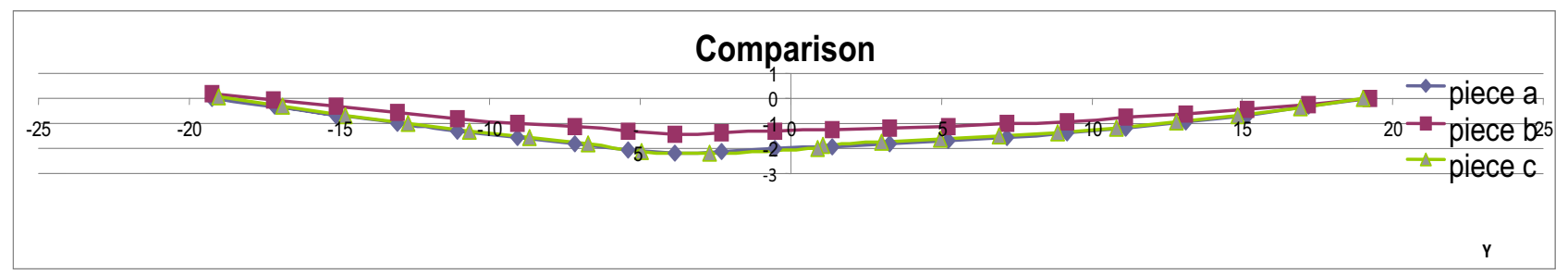

Figure 7. Specimen deformation analysis

We can observe that the minimum amplitude of deformed shape deviation is obtained in the case of the piece $\boldsymbol{b}$ (figure 7).

The results obtained with the device previously presented confirm the validity of the proposed new method.

The new methodology proposed has the following characteristics:

- high degree of generality by allowing only the adaptation of the macroelements dimensions used for the reduced order model of the forming process of complex parts;

- combining multiple process control techniques;

- use multiple active drawbeds differentiated in different segments of the part contour;

- optimal reference identification for programmed parameter, $h_{D B}$, of the process considering its effect on the stress rate $\sigma_{22} / \sigma_{11}$, the ideal feedback information for in-process control;

- continuous updating of the reference trajectory and control model using the database.

These characteristics provide the following advantages of the proposed method:

- reducing the time required for the process and control system design in case of complex deep-drawn parts;

- increased precision of metal forming process control by using the ratio $\sigma_{22} / \sigma_{11}$ as criterion for obtaining the reference trajectory parameter monitored $h_{D B}$;

- reducing costs represented by die try out stage using reduced order model of the process.

\section{References}

1. Y. Lim, R. Venugopal, G.A. Ulsoy, Proc. of the $17^{\text {th }}$ Int. Federation of Automatic Control World Congress, 1875 (2008).

2. H. Hayashi, H. Nishimura, The Annals of Dunarea de Jos University of Galati, Fasc. V, 3 (2009).

3. C.W. Hsu, A.G. Ulsoy, M.Y. Demeri, ASME J. Manuf. Sci. Eng. 122, 717 (2000).

4. C.W. Hsu, A.G. Ulsoy, M.Y. Demeri, J. of Mater. Proc. Tech. 127, 361 (2002).

5. V. Viswanathan, B. Kinsey, J. Cao, J Eng Mater-T ASME, 141 (2003).

6. M.H.A. Bonte, A.H. van den Boogaard, Int. J. Forming Proc., 1 (2005).

7. C. Maier, Al. Epureanu, V. Marinescu, V. Paunoiu, M. Afteni, F.B. Marin. Proc. of ModTech Int. Conf., 363 (2010).
8. C. Maier, Al. Epureanu, V. Marinescu, F.B. Marin, 6th Int. Conf. on Advanced in Dynamical Systems and Control, 133 (2010).

9. C. Maier, Al. Epureanu, F.B. Marin, J. of Engineering and Architecture 1, 1 (2013).

10. C. Maier, V. Paunoiu, V. Marinescu, The Annals of Dunarea de Jos University of Galati, Fasc. V, 25 (2013).

11. Michler J.R., Weinmann K.J., Kashani A.R., Majlessi S.A., J. of Mater. Proc. 43, 177 (1994).

12. A. Papaioanu, R. Schleich, M. Liewald, $7^{\text {th }}$ European LS-DYNA Conference (2009).

13. A. Papaioanu, M. Liewald, J. Mater. Form. 3, 155 (2010).

14. M. Samuel, J. of Mater. Proc. Tech. 122, 94 (2002).

15. L.R. Sanchez, Int. J. Mech. Sciences 42, 705 (2000).

16. D. Vlahovic, M. Liewald, $7^{\text {th }}$ International Stuttgart Symposium "Automotive and Engine Technology", (2007).

17. D. Vlahovic, M. Liewald, Proc. of $11^{\text {th }}$ ESAFORM International Conference, MS03, 175 (2008).

18. M. Liewald, D. Vlahovic, Patent No. DE 102006 047484 Al (2006). 